\title{
Frontal aslant tract projections to the inferior frontal gyrus
}

T. Szmuda ${ }^{1}$, M. Rogowska², P. Słoniewski ${ }^{1}$, A. Abuhaimed ${ }^{2}$, M. Szmuda ${ }^{3}$, J. Springer ${ }^{4}$, A. Sabisz ${ }^{5}$, J. Dzierżanowski ${ }^{1}$, A. Starzyńska ${ }^{6}$, T. Przewoźny $^{7}$

${ }^{1}$ Neurosurgery Department, Medical University of Gdansk, Poland

${ }^{2}$ Students Scientific Association, Neurosurgery Department, Medical University of Gdansk, Poland

${ }^{3}$ Neurology Department, Medical University of Gdansk, Poland

${ }^{4}$ Department of Preventive Medicine and Education, Medical University of Gdansk, Poland

${ }^{5}$ Department of Radiology, Medical University of Gdansk, Poland

${ }^{6}$ Department of Orofacial and Dental Surgery, Medical University of Gdansk, Poland

${ }^{7}$ Department of Otolaryngology, Medical University of Gdansk, Poland

[Received: 15 January 2017; Accepted: 11 March 2017]

Background: Frontal aslant tract (FAT) is a white matter bundle connecting the pre-supplementary motor area (pre-SMA) and the supplementary motor area (SMA) with the inferior frontal gyrus (IFG). The purpose of the present study was to evaluate the anatomical variability of FAT.

Materials and methods: Total number of fibres and the lateralisation index (LI) were calculated. We attempted to find factors contributing to the diversity of FAT regarding IFG terminations to the pars opercularis (IFG-Op) and to the pars triangularis (IFG-Tr). Magnetic resonance imaging of adult patients with diffusion tensor imaging (DTI) with total number of 98 hemispheres composed a cohort. V-shaped operculum was the most common (60.5\%).

Results: Total number of FAT fibres had widespread and unimodal distribution (6 to 1765; median: 160). Left lateralisation was noted in $64.3 \%$ of cases and was positively correlated with total number of FAT fibres and the bundle projecting to IFG-Op $(p<0.01)$. LI correlated with total number of FAT fibres ( $r=0.43$, $p<0.01)$. FAT projected predominantly to IFG-Op (88.9\%; 88 of 99). Only in 3 (3.1\%) cases more fibres terminated in IFG-Tr than in IFG-Op. Total number of FAT fibres and number of fibres terminating at IFG-Op did not correlate with the ratio of fibre numbers: FAT/IFG-Op, FAT/IFG-Tr and IFG-Op/IFG-Tr ( $p>0.05)$. The greater total number of fibres to IFG-Tr was, the higher were the ratios of IFG-Tr/ IFAT $(r=0.57, p<0.01)$ and IFG-Tr/IFG-Op $(r=0.32, p=0.04)$.

Conclusions: Among the IFG, the major termination of FAT is IFG-Op. Whereas the IFG-Tr projection seems to be related to the expansion of the entire FAT bundle regardless of side, domination and handedness. Nevertheless, FAT features a significant anatomical variability which cannot be explained in terms of DTI findings. (Folia Morphol 2017; 76, 4: 574-581)

Key words: white matter, frontal aslant tract, operculum, frontal lobe, diffusion tensor imaging, diffusion tensor tractography 


\section{INTRODUCTION}

Frontal aslant tract (FAT) is a recently described white matter pathway of the frontal lobe of the human brain $[3-5,9,12,15,16,19,20]$. FAT's name is derived from its oblique shape and it consists of short association connections between the posterior part of the inferior frontal gyrus (IFG) and the posterior region of the superior frontal gyrus (SFG) [5]. Diffusion tractography imaging (DTI) showed that FAT connects pre-supplementary/supplementary motor area (pre-SMA/SMA) with Broca's region, corresponding to the Brodmann areas 8.9 and 44.45 , respectively [3-5, 17, 19]. Although FAT has been identified in both hemispheres, a left lateralisation has been noted [4]. Moreover, most FAT fibres originate at pars opercularis (IFG-Op) of the IFG which can explain its functional role in the language model, supported by mapping techniques $[4,5]$. In dominant hemisphere FAT is associated with the initiation of speech and the fluency $[9,12]$. Speech arrest was observed in frontal lobe tumours infiltrating FAT as well as during its intraoperative electrical stimulation in awake surgeries $[11,16]$. FAT disconnection is attributed to stuttering, progressive aphasia or the development of Fox-Chavany-Marie syndrome [5, 13]. All of the above functions of FAT can be explained in terms of its connection to Broca's area which predominantly occupies IFG-Op [18]. However, some projections of FAT may reach pars triangularis (IFG-Tr), pars orbitalis (IFG-Or) or pre-central gyrus (PrCG) [4]. To date, IFG projections of FAT has not been scrutinised.

The aim of our study is to more accurately describe the anatomical variability of FAT in terms of the number of fibres, lateralisation and projection to IFG. We attempt to find the factors contributing to the diversity of FAT. In the context of increasing knowledge of the functional role of FAT, the anatomical background should be provided beforehand. By demonstrating the irregularity of FAT we strengthen the view that FAT is not an isomorphic structure.

\section{MATERIALS AND METHODS}

\section{Participants}

This study's participants were 54 adult patients (24 males and 30 females) who qualified for surgery at the Neurosurgery Department, Medical University of Gdansk from 2010 to 2015. Patients with any pathological lesions potentially interfering with regions of interest (Broca's and pre-SMA/SMA) and the track of FAT bundle were consecutively excluded; these lesions included tumour, oedema and previous frontal lobe surgery. The mean age of patients was 49.5 years (min-max: 21-80; standard deviation [SD] \pm 49.5). Handedness was determined (right/left $=54 / 3$ ). Patients with aphasia were excluded.

Informed consent was obtained from all participants and the study protocol was approved by the local Ethics Committee. The project was supported by grant no. 01-0056/08.

\section{Image acquisition}

The magnetic resonance imaging (MRI) scans of the human brain (T1-, T2-weighed and DTI with single-shot echo-planar imaging) were acquired using a 20-chanel head/neck coil on a 1.5 T Siemens Magnetom Area scanner (Erlangen, Germany). We used three repetitions of 20 non-collinear diffusion gradient directions for contiguous slices. They were all acquired parallel to the anterior-posterior commissure line. DTI acquisition parameters were: $5.0 \mathrm{~mm}$ slice thickness, $128 \times 128$ matrix, $240 \mathrm{~mm} \times 240 \mathrm{~mm}$ field of view, $b=1000 \mathrm{~s} / \mathrm{m}^{2}$, repetition time $=3500 \mathrm{~ms}$, echo time $=83.0 \mathrm{~ms}$ and weak fat suppression. DTI output volumes were registered together with T1 sequence for anatomical correspondence. The calculation included fractional anisotropy (FA), mean diffusivity (MD), directionally encoded colours (DEC), apparent diffusion coefficient (ADC).

\section{Procedure}

All measurements of FAT were performed, refined and depicted using StealthViz neuronavigation software (Medtronic Inc., Minneapolis, USA) with the StealthDTI module. Using the option of segmentation, the regions of interest (SMA and pre-SMA, pars opercularis of the IFG [IFG-Op], pars triangularis of the IFG [IFG-Tr] and pars orbitalis of the IFG) were identified and manually marked (Fig. 1).

Then, the ipsilateral neural bundles between particular parts of SFG and IFG were automatically plotted by the software. Seed density was set at 2.0, the maximal directional change at 50 degrees, and the minimal fibre length at $1 \mathrm{~mm}$. All DTI tracking settings were consistent throughout the MRls. Lateralisation index was calculated by the established formula $[4,14]$.

The anatomical variability of IFG-Tr was evaluated in T1/T2-weighed MRI. Depending on the configuration of the Sylvian fissure, the IFG-Tr can form a V-shape (two rami spread from one point right above the 


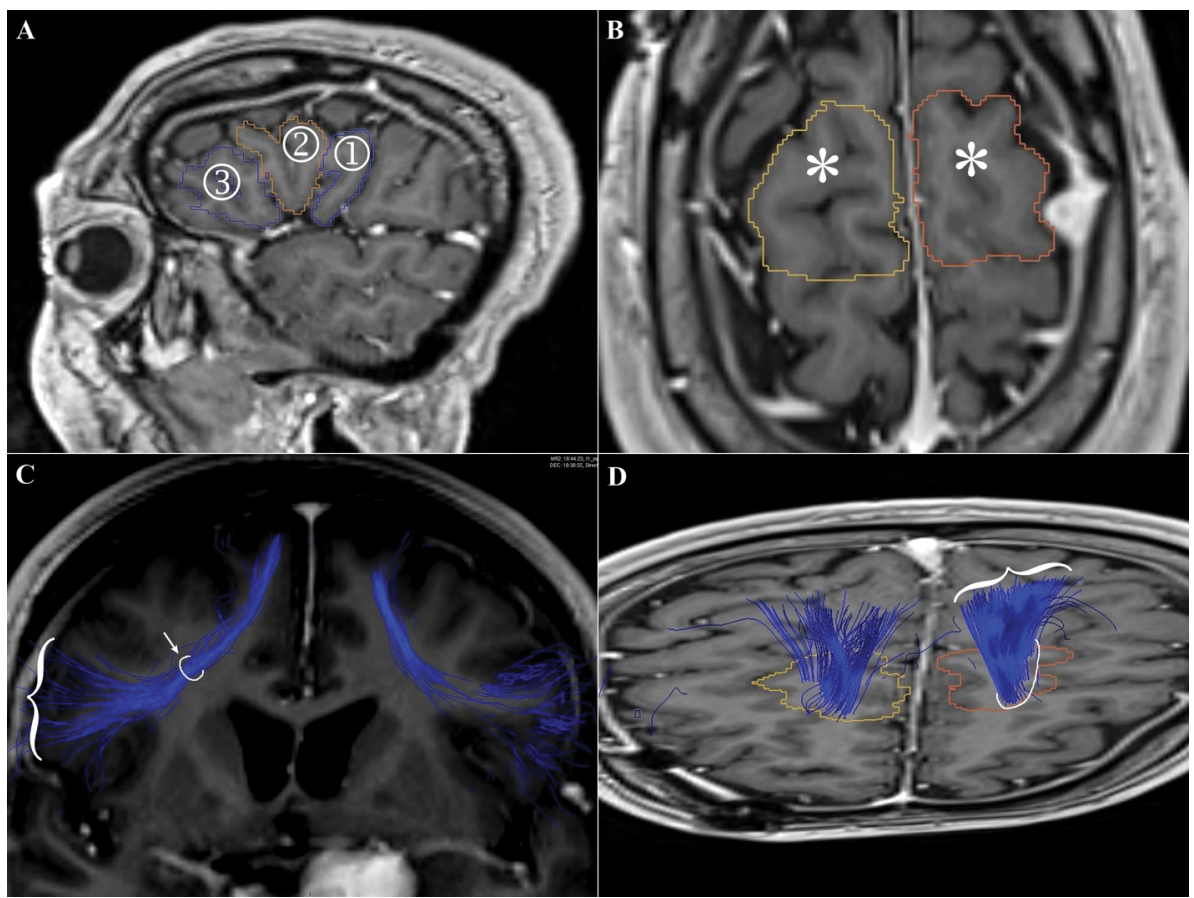

Figure 1. Method of tracking the frontal aslant tract (FAT); A. Region of interests of operculum of inferior frontal gyrus (IFG) were anatomically segmented: 1 - pars opercularis; 2 - pars triangularis; 3 - pars orbitalis; B. Pre-supplementary and supplementary motor area (pre-SMA/ /SMA) were segmented from the IFG and marked with an asterisk $\left({ }^{*}\right)$; C. FAT (blue bundle) was tracked from the SMA to the operculum. A narrow bundle of packed fibres was marked with an arrow. Curly bracket " $\{$ " is shown to emphasize the widespread propagation of FAT in IFG; D. FAT (blue bundle) ending in the superior frontal gyrus. Curly bracket " $\{$ " demonstrates a concentrated projection of FAT to the pre-SMA/SMA.

Sylvian fissure that forms the apex of the triangle), a U-shape (mentioned rami spread from one point like in V-shape, but further apart), a Y-shape (rami spread from common stem) and a J-shape (an asymmetry in length of two rami) (Fig. 2) [7].

\section{Statistical analyses}

Mean values, standard deviation (SD) and ranges were analysed. Constant variables were compared by means of $\mathrm{t}$-test (for independent means, normal distribution) or Mann-Whitney test (unless normal distribution of data, Shapiro-Wilk [S-W] test). Statistica v.12.0 (StatSoft, Tulsa Inc., USA) and GraphPad Prism v.6.05 (GraphPad Company, La Jolla, CA) were used for statistical analyses; the statistical significance level $(p)$ was set at 0.05 .

\section{RESULTS}

\section{The shape of IFG}

The V- $(60.5 \% ; n=69)$ and $Y-(17.5 \% ; n=20)$ shapes were the most common shapes of the operculum. $8.7 \%(n=10)$ and $1.7 \%(n=2)$ were $U$ - and $\mathrm{J}$-shaped, respectively, whereas in 13 hemispheres the operculum could not be attributed to any of the pre-defined shapes.

\section{Number of fibres}

No connections between pre-SMA or SMA and pars orbicularis of IFG were identified in any of the patients. The following analysis of FAT focused on entire FAT bundle and its two subdivisions: fibres terminating at IFG-Op and IFG-Tr.

Non-normal distributions (S-W test, $p<0.05$ ) were observed in the total number of fibres of the FAT bundle, fibres projecting to IFG-Op and IFG-Tr. The total number FAT fibres varied between 6 and 1765 (median: 160). Mean number of FAT fibres was $270.2 \pm 299.9$. Standard deviation greater than the mean as well as greater mean than median were also noted for the total number of fibres to either IFG-Op or IFG-Tr (Table 1).

Unimodal and asymmetrical distribution was demonstrated while plotting total number of fibres (FAT and its subdivisions) (Fig. 3).

Skewness exceeding 1 (as a measure of strong asymmetrical distribution) was observed in the entire 


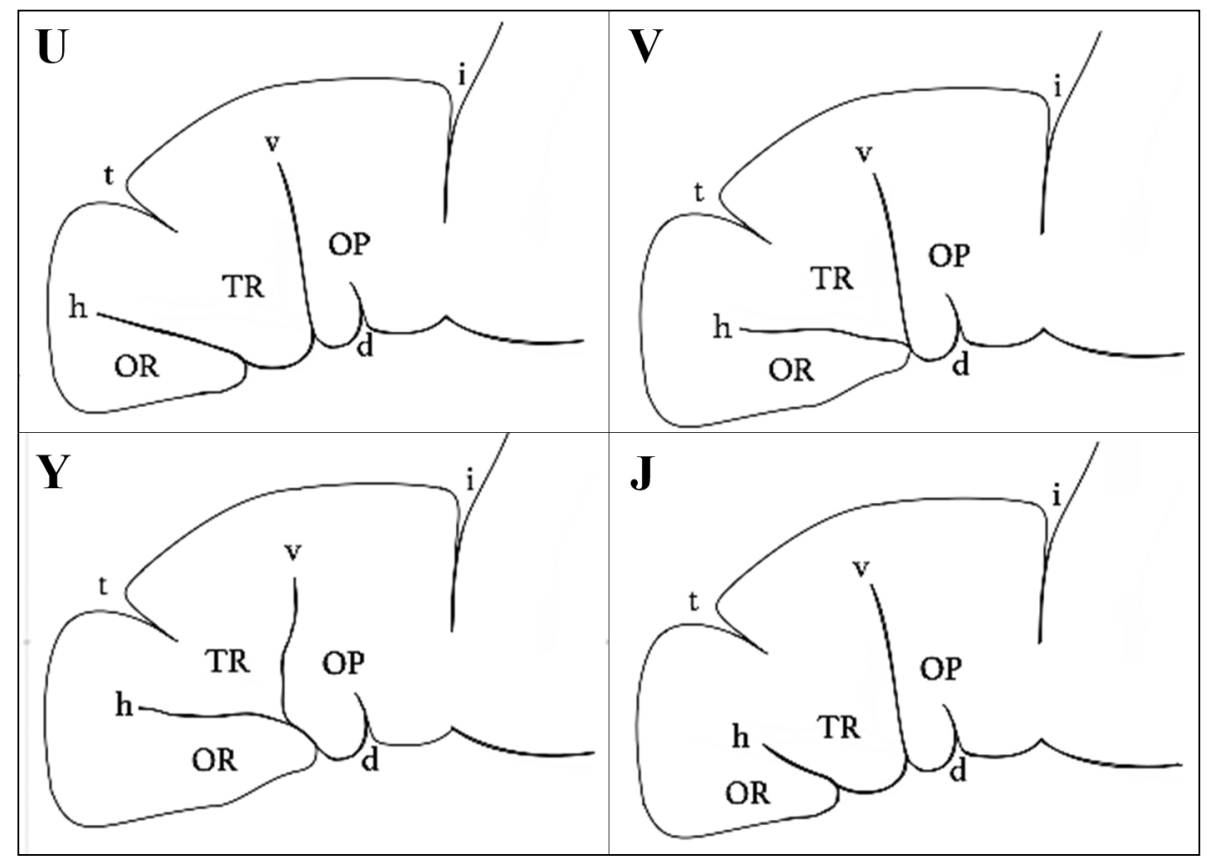

Figure 2. Anatomical variability of pars triangularis; U. U-shaped; V. V-shaped; Y. Y-shaped; J. J-shaped pars triangularis; d - diagonal sulcus; $\mathrm{h}$ - horizontal limb of the Sylvian fissure; $\mathrm{i}$ — inferior precentral sulcus; $\mathrm{OP}$ - pars opercularis; $\mathrm{OR}$ — pars orbitalis; $\mathrm{t}$ - sulcus triangularis; TR — pars triangularis; $v$ — vertical (ascending) limb the of Sylvian fissure.

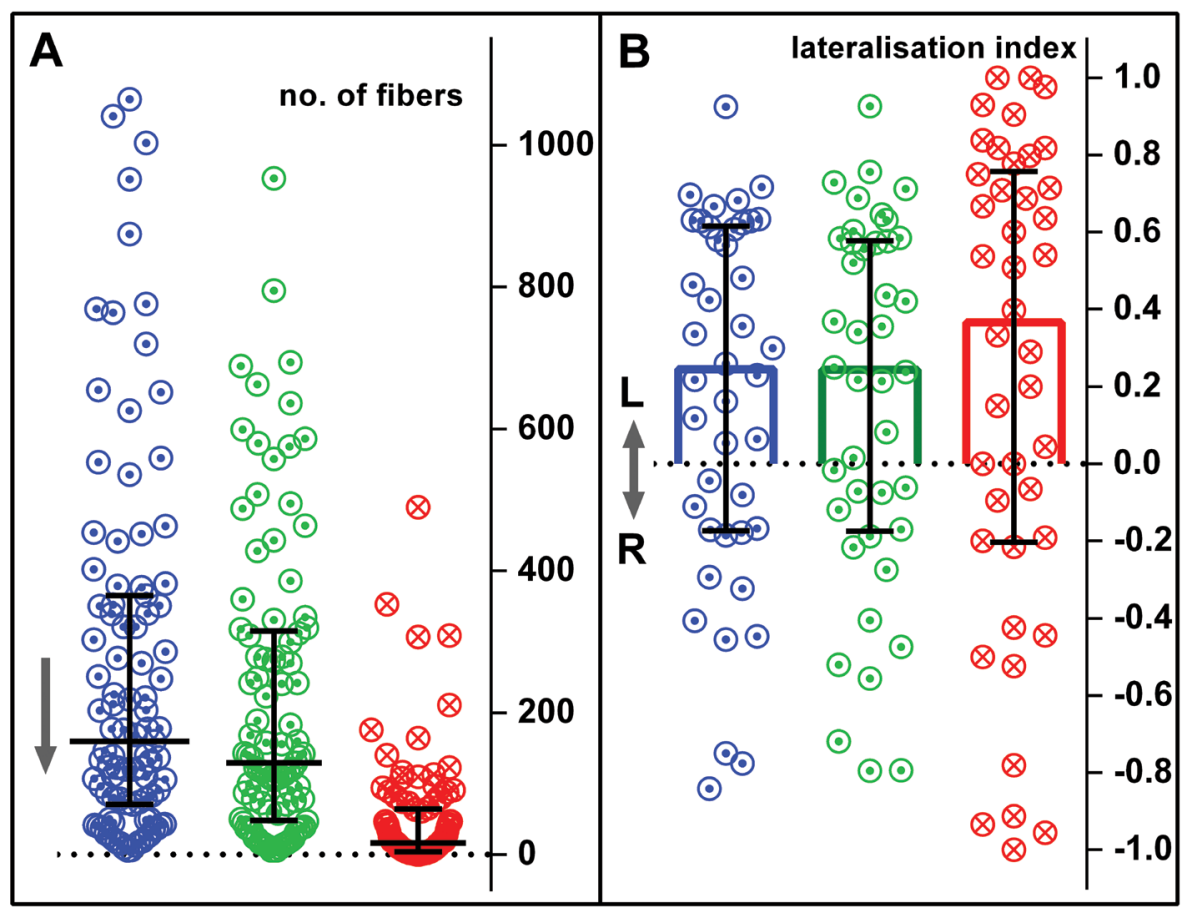

Figure 3. Visual presentation of the number of fibres (A) and the lateralisation index (LI) (B) of the entire frontal aslant tract (FAT) pathway (blue dots), subdivision of FAT projecting to the pars opercularis (green dots) and to the pars triangularis (red dots). The black line (A) and the upper side of the rectangle (B) demonstrate the median value. The width between the whiskers (either $\mathbf{A}$ or $\mathbf{B}$ ) denotes the interquartile range. Grey arrow (A) points to the left-sided concentration of the observations. The double-sided arrow (B) shows the direction of the left (positive values of $\mathrm{LI}$ ) and the right (negative values of LI) lateralisation. 
Table 1. The distribution of the number of frontal aslant tract (FAT) fibres and its subdivisions

\begin{tabular}{ccccccc}
\hline & Mean & Standard deviation & Min-max & Median & Skewness & Deviation of skewness \\
\hline No of fibres: & & & & & \\
Entire FAT & 270.2 & 299.9 & $6-1765$ & 160.0 & 2.12 & 0.24 \\
To IFG-Op & 225.0 & 244.8 & $6-1458$ & 129.0 & 2.08 & 0.24 \\
To IFG-Tr & 48.2 & 79.7 & $0-490$ & 16.5 & 3.21 & 0.24 \\
Ll of FAT bundle & 0.18 & 0.46 & $-0.84-0.93$ & 0.24 & NA & NA \\
Ll of IFG-Op & 0.17 & 0.47 & $-0.79-0.93$ & 0.24 & NA & NA \\
Ll of IFG-Tr & 0.22 & 0.62 & $-1.00-1.00$ & 0.37 & NA & NA \\
\hline
\end{tabular}

IFG-Op — pars opercularis of the inferior frontal gyrus; IFG-Tr — pars triangularis of the inferior frontal gyrus; LI — lateralisation index; NA — not applicable

Table 2. The ratio of number of fibres: ending in pars opercularis (IFG-Op) of the inferior frontal gyrus, pars triangularis (IFG-Tr) of the inferior frontal gyrus and the frontal aslant tract. Numbers in bold indicate the most frequent observations

\begin{tabular}{lccc}
\hline $\begin{array}{l}\text { Quotient of the number } \\
\text { of fibres (1) to (2) }\end{array}$ & \multicolumn{3}{c}{$\mathbf{N}$ (\% of column) } \\
\cline { 2 - 4 } & (1) IFG-0p / (2) entire FAT & (1) IFG-Tr / (2) entire FAT & (1) IFG-Tr / (2) IFG-0p \\
\hline $0.0-0.2$ & $2(2.0)$ & $\mathbf{4 5}(45.5)$ & $\mathbf{8 3}(\mathbf{8 5 . 6 )}$ \\
$0.2-0.4$ & $1(1.0)$ & $\mathbf{3 8}(38.4)$ & $13(13.4)$ \\
$0.4-0.6$ & $8(8.1)$ & $13(13.1)$ & $0(0.0)$ \\
$0.6-0.8$ & $\mathbf{4 0 ( 4 0 . 4 )}$ & $2(2.0)$ & $0(0.0)$ \\
$>0.8$ & $\mathbf{4 8}(48.5)$ & $1(1.0)$ & $1(1.0)$ \\
\hline
\end{tabular}

FAT bundle distribution and its subdivisions. This means that most of the obtained number of fibres was low with some outliers and/or extreme values (Table 1).

\section{Lateralisation index (LI)}

Left lateralisation of FAT was observed in $64.3 \%$ of MRIs (27 of 42). LI of the entire FAT bundle and the subdivisions (to IFG-Op and IFG-Tr) varied across the cases (see the distribution of $\mathrm{LI}$ in Fig. 3) despite that the mean LI was greater than 0 (left lateralisation). Neither the patient's handedness ( $p=$ $=0.55)$, sex $(p=0.08)$, age $(p=0.78)$ or the shape of the operculum $(p=0.65$ ) influenced $\mathrm{LI}$ of the FAT bundle. The left lateralisation was related to the increased number of FAT fibres and its subdivisions ending up in the IFG-Op $(p<0.01)$. The total number of FAT fibres correlated positively with the LI $(r=0.43, p<0.01)$.

\section{FAT projections}

In most cases the FAT projected to the IFG-Op (88.9\%; 88 of 99). More than $60 \%$ of FAT fibres ended up in the IFG-Tr only in 3 of the 98 hemispheres ana- lysed (3.1\%). A greater number of fibres terminated at the IFG-Tr than at the IFG-Op in only one case; a left-handed patient without any obvious speech disturbances (right hemisphere with a Y-shaped operculum) (Table 2).

Factors influencing the variety of FAT projections to the IFG were analysed. Neither the total number of FAT fibres nor the number of fibres terminating at IFG-Op correlated with the ratio of fibres: FAT/ /IFG-Op, FAT/IFG-Tr and IFG-Op/IFG-Tr ( $p>0.05$ ). On the other hand, the total number of fibres projecting to the IFG-Tr was positively correlated with the ratios of IFG-Tr/FAT $(r=0.57, p<0.01)$ and IFG-Tr/IFG-Op $(r=0.32, p=0.04)$ (Table 3$)$.

\section{DISCUSSION}

Although little is known about the functional properties of the FAT, there is a growing interest in that novel tract $[3,9,12,15,16,19,20]$. The functional roles of FAT can be scrutinised to motor functions and language processing. Regarding motor control, loss of FAT integration can lead to a rare suprabulbar palsy (Foix-Chavany-Marie syndrome) affecting the orofacial muscles [13]. This syndrome 
Table 3. Number of fibres, lateralisation index and ratio of number of frontal aslant tract (FAT) fibres and its subdivisions between various factors: shape of operculum, sex, side, lateralisation and handedness

\begin{tabular}{|c|c|c|c|c|c|c|c|c|c|c|}
\hline Neural bundle & \multicolumn{2}{|c|}{ U, J or Y shape } & \multicolumn{2}{|c|}{ V shape } & $\mathbf{p}$ & \multicolumn{2}{|c|}{ Female sex } & \multicolumn{2}{|c|}{ Male sex } & $\mathbf{p}$ \\
\hline Whole FAT & \multicolumn{2}{|c|}{$202.9 \pm 215.4$} & \multicolumn{2}{|c|}{$300.8 \pm 328.2$} & 0.13 & \multicolumn{2}{|c|}{$315.2 \pm 361.9$} & \multicolumn{2}{|c|}{$234.3 \pm 236.5$} & 0.18 \\
\hline IFG-Op & \multicolumn{2}{|c|}{$177.3 \pm 191.5$} & \multicolumn{2}{|c|}{$246.4 \pm 263.7$} & 0.20 & \multicolumn{2}{|c|}{$260.9 \pm 298.3$} & \multicolumn{2}{|c|}{$196.4 \pm 190.1$} & 0.20 \\
\hline IFG-Tr & \multicolumn{2}{|c|}{$28.1 \pm 44.7$} & \multicolumn{2}{|c|}{$56.6 \pm 90.4$} & 0.10 & \multicolumn{2}{|c|}{$57.8 \pm 93.1$} & \multicolumn{2}{|c|}{$39.6 \pm 67.3$} & 0.26 \\
\hline \multicolumn{11}{|c|}{ Lateralisation index } \\
\hline Whole FAT & \multicolumn{2}{|c|}{$0.21 \pm 0.47$} & \multicolumn{2}{|c|}{$0.17 \pm 0.47$} & 0.75 & \multicolumn{2}{|c|}{$0.32 \pm 0.46$} & \multicolumn{2}{|c|}{$0.07 \pm 0.44$} & 0.08 \\
\hline IFG-Op & \multicolumn{2}{|c|}{$0.22 \pm 0.52$} & \multicolumn{2}{|c|}{$0.15 \pm 0.45$} & 0.66 & \multicolumn{2}{|c|}{$0.28 \pm 0.48$} & \multicolumn{2}{|c|}{$0.09 \pm 0.45$} & 0.19 \\
\hline IFG-Tr & \multicolumn{2}{|c|}{$0.15 \pm 0.57$} & \multicolumn{2}{|c|}{$0.26 \pm 0.64$} & 0.62 & \multicolumn{2}{|c|}{$0.44 \pm 0.56$} & \multicolumn{2}{|c|}{$0.06 \pm 0.62$} & 0.05 \\
\hline \multicolumn{11}{|c|}{ Ratio (expressed in \%) } \\
\hline IGF-Op/FAT & \multicolumn{2}{|c|}{$83.5 \pm 20.8$} & \multicolumn{2}{|c|}{$81.0 \pm 18.0$} & 0.54 & \multicolumn{2}{|c|}{$79.7 \pm 20.1$} & \multicolumn{2}{|c|}{$83.5 \pm 17.9$} & 0.32 \\
\hline IFG-Tr/FAT & $13.4 \pm 14$ & & & \pm 15.1 & 0.19 & $18.1 \pm$ & $=16.0$ & $14.8 \pm$ & 13.8 & 0.26 \\
\hline IFG-Tr/IFG-Op & $20.2 \pm 28$ & & & \pm 48.2 & 0.36 & $32.1 \pm$ & $=57.2$ & $21.8 \pm$ & 27.1 & 0.24 \\
\hline Neural bundle & Left side & & & $\mathbf{p}$ & $\begin{array}{c}\text { Left } \\
\text { lateralisation }\end{array}$ & $\begin{array}{c}\text { Right } \\
\text { lateralisation }\end{array}$ & $\mathbf{p}$ & $\begin{array}{c}\text { Right } \\
\text { handedness }\end{array}$ & $\begin{array}{c}\text { Left } \\
\text { handedness }\end{array}$ & p \\
\hline Whole FAT & $304.2 \pm 336.0$ & 225.9 & 240.0 & 0.20 & $407.4 \pm 321.7$ & $147.1 \pm 119.7$ & $<0.01$ & $272.1 \pm 302.8$ & $209.7 \pm 214.3$ & 0.72 \\
\hline IFG-Op & $251.4 \pm 281.3$ & 189.0 & 180.9 & 0.22 & $338.8 \pm 269.6$ & $129.1 \pm 113.3$ & $<0.01$ & $227.7 \pm 247.6$ & $142.0 \pm 121.8$ & 0.55 \\
\hline IFG-Tr & $52.8 \pm 82.3$ & 41.0 & 77.1 & 0.47 & $68.6 \pm 96.2$ & $18.0 \pm 27.4$ & 0.05 & $47.1 \pm 79.9$ & $67.7 \pm 94.0$ & 0.66 \\
\hline Ratio (expresse & & & & & & & & & & \\
\hline IGF-Op/FAT & $82.1 \pm 15.4$ & 81.5 & 22.8 & 0.87 & $84.3 \pm 11.6$ & $85.7 \pm 14.7$ & 0.74 & $81.9 \pm 19.1$ & $76.8 \pm 13.6$ & 0.64 \\
\hline IFG-Tr/FAT & $17.9 \pm 15.4$ & 14.1 & 13.9 & 0.21 & $15.7 \pm 11.5$ & $14.3 \pm 14.9$ & 0.74 & $16.0 \pm 14.9$ & $23.2 \pm 13.6$ & 0.41 \\
\hline IFG-Tr/IFG-Op & $29.9 \pm 51.6$ & 21.4 & 27.6 & 0.33 & $21.2 \pm 19.7$ & $21.1 \pm 27.3$ & 0.99 & $26.1 \pm 43.6$ & $33.3 \pm 26.3$ & 0.78 \\
\hline
\end{tabular}

IFG- $0 p$ - pars opercularis of the inferior frontal gyrus, IFG-Tr — pars triangularis of the inferior frontal gyrus

might be attributed to a motor subdivision of the FAT ending in the Brodmann Area 6. According to the present understanding of language processing, the FAT is involved in specific functions as it connects the IFG-Op (Broca's area) with the pre-SMA and the SMA $[8,11,18]$. Damage to the Broca's area of the linguistically dominant (usually left) hemisphere, has been called "Broca's aphasia" (or "non-fluent", "expressive" aphasia) [18]. The symptoms include the inability to produce grammatically correct and fluent speech with a preserved comprehension in most cases. On the other hand, damage to the pre-SMA causes a mild to severe speech impairment ranging from affected fluency to complete speech arrest (mutism) $[10-12,20]$. A recent study of patients suffering from primary progressive aphasia showed that pathological changes within the FAT affect verbal fluency with no influence on the overall language impairment, grammar deficits, repetition and single word comprehension [5]. The physiological roles of specific FAT subdivisions have not been fully revealed yet [16]. Lesions of the FAT bundle projecting to the pre-SMA resulted in loss of linguistic functions while the connections to the SMA were related to articulation and word production [1]. On the IFG side, FAT terminates at different parts of the operculum. Catani et al. [4] reported that the predominant part of the tract ends (or starts) in the IFG-Op, but some may reach IFG-Tr and pars orbitalis $[4,8,10]$. This illustrates the incompletely understood heterogeneity of the bundle.

To date, no studies focused on how many fibres selectively reach the IFG-Tr and the IFG-Op, and what affects this type of heterogeneity. The shapes of the IFG-Tr are defined by two rami of Sylvian fissure the anterior horizontal ramus and anterior ascending ramus. Superiorly, the triangle is limited by inferior frontal sulcus $[2,7]$. However, the two rami of the Sylvian fissure can form a V-, U-, Y- or occasionally a J-shape [7]. According to volumetric magnetic resonance methodologies, the volume of the IFG-Tr is generally greater in the left hemisphere of right-handed people, while the V-shape is the most common shape in both hemispheres and is associated with the largest 
surface area [7]. However, in our data the shape of the IFG-Tr did not influence the number of FAT fibres, the extent of subdivisions or any other factor.

On the other hand, the total number of fibres terminating at the IFG-Tr positively correlated with the ratio of number of the IFG-Tr fibres to the FAT fibres. In other words, if the FAT bundle was well-developed, the greater percentage of this bundle contributed the IFG-Tr projection. This may reflect that a more developed FAT has a different distribution of fibres than a smaller FAT bundle, regardless of hemisphere and handedness. The above novel observation remains unexplained and is difficult to discuss as not stated in the literature. Our study was the first to evaluate the number of FAT fibres dividing into subdivisions (to the IFG-Op and to the IFG-Tr). Furthermore, the next stage of FAT analysis should involve the size of the entire FAT and its distribution to the IFG-Op and the IFG-Tr.

One potent factor influencing FAT size is the lateralisation of human brain [4]. FAT was regarded as strongly lateralised, with left domination in right-handed people [6]. In the present analysis, despite disperse $\mathrm{LI}$ values, left lateralisation was noted in most cases (64.3\%). Mean LI was positive for the entire FAT, the IFG-Op and the IFG-Tr portions. Greater number of FAT fibres was observed for left lateralised brains and for higher $\mathrm{LI}$ values regardless of the side of the brain. One of the conclusions drawn from the above finding is that lateralisation plays a crucial role for the FAT development, opposite to the hemisphere and handedness.

Considering various number of FAT fibres demonstrated in our study, the puzzling irregularity of FAT underlie the inter-individual (a case differing from each other) or intra-individual variability (differences in DTI acquisition, processing and FAT tracking). Although the DTI studies could be supported by the data from the functional MRIs, a handful of clinical studies with intraoperative stimulation and monitoring confirm that reactions to FAT compromise might be different $[3,8,10,13,15-17,19]$.

\section{CONCLUSIONS}

The clinical significance of our study is that the FAT should be added to the list of 'dangerous areas' of the frontal lobe in modern neurosurgical DTI planning, parallel to Broca's area.

This study was supported by the Ministry of Science and Higher Education of the Republic of Poland, a grant for young scientists (no. 01-0056/08).

\section{REFERENCES}

1. Alario FX, Chainay $H$, Lehericy $S$, et al. The role of the supplementary motor area (SMA) in word production. Brain Res. 2006; 1076(1): 129-143, doi: 10.1016/j.brainres.2005.11.104, indexed in Pubmed: 16480694.

2. Ayberk G, Yagli OE, Comert A, et al. Anatomic relationship between the anterior sylvian point and the pars triangularis. Clin Anat. 2012; 25(4): 429-436, doi: 10.1002/ /ca.21264, indexed in Pubmed: 22488994.

3. Broce I, Bernal B, Altman N, et al. Fiber tracking of the frontal aslant tract and subcomponents of the arcuate fasciculus in 5-8-year-olds: Relation to speech and language function. Brain Lang. 2015; 149: 66-76, doi: 10.1016/j. bandl.2015.06.006, indexed in Pubmed: 26186231.

4. Catani M, Dell'acqua F, Vergani F, et al. Short frontal lobe connections of the human brain. Cortex. 2012; 48(2): 273-291, doi:10.1016/j.cortex.2011.12.001, indexed in Pubmed: 22209688.

5. Catani M, Mesulam MM, Jakobsen E, et al. A novel frontal pathway underlies verbal fluency in primary progressive aphasia. Brain. 2013; 136(Pt 8): 2619-2628, doi: 10.1093/ /brain/awt163, indexed in Pubmed: 23820597.

6. Dhakar MB, llyas $\mathrm{M}$, Jeong JW, et al. Frontal aslant tract abnormality on diffusion tensor imaging in an aphasic patient with 49, XXXXY syndrome. Pediatr Neurol. 2016; 55: 64-67, doi: 10.1016/j.pediatrneurol.2015.10.020, indexed in Pubmed: 26706051.

7. Foundas AL, Weisberg A, Browning CA, et al. Morphology of the frontal operculum: a volumetric magnetic resonance imaging study of the pars triangularis. J Neuroimaging. 2001; 11(2): 153-159, indexed in Pubmed: 11296585.

8. Fujii M, Maesawa $S$, Ishiai $S$, et al. Neural Basis of Language: An Overview of An Evolving Model. Neurol Med Chir (Tokyo). 2016; 56(7): 379-386, doi:10.2176/nmc. ra.2016-0014, indexed in Pubmed: 27087195.

9. Fujii M, Maesawa $S$, Motomura $K$, et al. Intraoperative subcortical mapping of a language-associated deep frontal tract connecting the superior frontal gyrus to Broca's area in the dominant hemisphere of patients with glioma. J Neurosurg. 2015; 122(6): 1390-1396, doi:10.3171/2014.10. JNS14945, indexed in Pubmed: 25816090.

10. Kemerdere R, de Champfleur NM, Deverdun J, et al. Role of the left frontal aslant tract in stuttering: a brain stimulation and tractographic study. J Neurol. 2016; 263(1): 157-167, doi: 10.1007/s00415-015-7949-3, indexed in Pubmed: 26559819.

11. Kinoshita M, de Champfleur NM, Deverdun J, et al. Role of fronto-striatal tract and frontal aslant tract in movement and speech: an axonal mapping study. Brain Struct Funct. 2015; 220(6): 3399-3412, doi: 10.1007/s00429-014-0863-0, indexed in Pubmed: 25086832.

12. Kronfeld-Duenias V, Amir O, Ezrati-Vinacour R, et al. The frontal aslant tract underlies speech fluency in persistent developmental stuttering. Brain Struct Funct. 2016; 221(1): 365-381, doi: 10.1007/s00429-014-0912-8, indexed in Pubmed: 25344925.

13. Martino J, de Lucas EM, Ibáñez-Plágaro FJ, et al. FoixChavany-Marie syndrome caused by a disconnection between the right pars opercularis of the inferior frontal gyrus and the supplementary motor area. J Neurosurg. 
2012; 117(5): 844-850, doi: 10.3171/2012.7.JNS12404, indexed in Pubmed:22957529.

14. Qiu D, Tan LH, Siok WT, et al. Lateralization of the arcuate fasciculus and its differential correlation with reading ability between young learners and experienced readers: a diffusion tensor tractography study in a Chinese cohort. Hum Brain Mapp. 2011; 32(12): 2054-2063, doi: 10.1002/ /hbm.21168, indexed in Pubmed: 21259386.

15. Rizio AA, Diaz MT. Language, aging, and cognition: frontal aslant tract and superior longitudinal fasciculus contribute toward working memory performance in older adults. Neuroreport. 2016; 27(9): 689-693, doi: 10.1097/WNR.0000000000000597, indexed in Pubmed: 27138951.

16. Sierpowska J, Gabarrós A, Fernandez-Coello A, et al. Morphological derivation overflow as a result of disruption of the left frontal aslant white matter tract. Brain Lang. 2015; 142: 54-64, doi: 10.1016/j.bandl.2015.01.005, indexed in Pubmed: 25658634.
17. Tanaka N, Grant PE, Suzuki N, et al. Multimodal imaging of spike propagation: a technical case report. AJNR Am J Neuroradiol. 2012; 33(6): E82-E84, doi: 10.3174/ajnr. A2701, indexed in Pubmed: 21960488.

18. Tremblay P, Dick AS. Broca and Wernicke are dead, or moving past the classic model of language neurobiology. Brain Lang. 2016; 162: 60-71, doi: 10.1016/j. bandl.2016.08.004, indexed in Pubmed: 27584714.

19. Vassal F, Boutet C, Lemaire JJ, et al. New insights into the functional significance of the frontal aslant tract: an anatomo-functional study using intraoperative electrical stimulations combined with diffusion tensor imaging-based fiber tracking. Br J Neurosurg. 2014; 28(5): 685-687, doi: 10.3109/ /02688697.2014.889810, indexed in Pubmed: 24552256.

20. Vergani F, Lacerda L, Martino J, et al. White matter connections of the supplementary motor area in humans. J Neurol Neurosurg Psychiatry. 2014; 85(12): 1377-1385, doi: 10.1136/jnnp-2013-307492, indexed in Pubmed: 24741063. 